\title{
Perspectivas individuais sobre a necessidade de mudança: estudo de caso na Indústria Têxtil e do Vestuário Portuguesa
}

\author{
Laura Costa Maia ${ }^{1}$, Anabela Alves ${ }^{1}$, Celina Pinto Leão ${ }^{2}$ \\ id1932@alunos.uminho.pt, anabela@dps.uminho.pt, celina.p.leao@algoritmi.uminho.pt \\ ${ }^{1}$ Centro de Gestão Industrial e da Tecnologia (CGIT), Escola de Engenharia da Universidade do Minho, \\ Departamento de Produção e Sistemas, Campus de Azurém, 4804 - 533 Guimarães, Portugal \\ ${ }^{2}$ Centro Algoritmi, Escola de Engenharia da Universidade do Minho, Departamento de Produção e Sistemas, \\ Campus de Azurém, 4804 - 533 Guimarães, Portugal
}

DOI: 10.17013/risti.e2.115-127

\begin{abstract}
Resumo: O objectivo deste trabalho é perceber de uma forma individual a necessidade de mudança e as suas causas, num estudo de caso na Indústria Têxtil e do Vestuário. Para atingir este objetivo, foram realizadas várias entrevistas semiestruturadas a chefias de nível hierárquico médio/alto da empresa. Foi possível perceber como os trabalhadores pretendem intervir na mudança bem como as suas atitudes nessa mudança. Para além destes tópicos, as estratégias usadas para participar na mudança, o porquê e como fazer essa mudança, alternativas e ganhos previstos foram também discutidos. Os resultados deste trabalho revelam uma consciencialização dos trabalhadores à necessidade da mudança e fatores como motivação, envolvimento, informação adaptação, foram identificados como importantes para a mudança. Risco e instabilidade foram fatores identificados como os mais negativos. E esta necessidade de mudança indispensável para a implementação de uma estratégia Lean Production bemsucedida na empresa.
\end{abstract}

Palavras-chave: Análise Qualitativa; Entrevistas semiestruturadas; Indústria Têxtil e do Vestuário; Lean Production.

Individual perspectives on the need for change: a case study in the Portuguese Textile and Clothing Industry

Abstract: The objective of this research is to study individual understandings regarding the need for change and its causes in a Textile and Clothing Industry (TCI) case study. To achieve this objective, semi-structured interviews with managers of medium/high hierarchical company's level were conducted. The results reveal how workers intend to intervene in the change and their attitudes on that change. Topics as characterization of the working population; awareness of the need for change and its causes; strategies used to participate on the change; why and how to make the change; expected outcomes/results, were also addressed. The findings of this study reveal an awareness of workers to the need 
for change and factors such as motivation, involvement, information, and adaptation were identified as important to the change. Instability and risk were identified as the most negative factors. This need to change it is essential to successfully implement a Lean Production strategy in a company.

Keywords: Interviews; Lean Production; Qualitative analysis; Textile and Clothing Industry.

\section{Introdução}

A Indústria Têxtil e do Vestuário (ITV) tem um papel importante na economia nacional Portuguesa sendo uma das poucas indústrias que contribui positivamente para a balança comercial, com impacto significativo sobre o volume de emprego e redução das margens de comercialização. Esta indústria representa $9 \%$ das exportações nacionais (na década de 90, esse valor era de 30\%) (aicep, 2014). Está espalhada em todo o território nacional, embora concentrada em duas regiões principais: a região do Norte de Portugal, onde estão localizadas as empresas algodoeiras e, na região da Beira Interior, onde estão localizadas as empresas de lanifícios. A ITV é composta por dois grandes sectores: (1) o sector têxtil - que inclui a produção de fibras, fiação, tecelagem, malharia e acabamentos (tinturaria, estamparia e acabamentos) e (2) o sector do vestuário - que inclui confecção de vestuário e acessórios. A ITV é constituída principalmente por Pequenas e Médias Empresas (PME), com uma forte estrutura familiar tradicional (aicep, 2014).

Devido à sua natureza de processamento, a ITV é fortemente dependente de recursos naturais (água, fibras naturais, corantes, entre outros). Por exemplo, os processos de tingimento e acabamento consomem elevadas quantidades de água e energia. Com a diminuição destes recursos naturais, esta indústria poderá enfrentar grandes dificuldades e desafios. Este sector da ITV é também fortemente influenciado pelas estações do ano, tendências e moda. Às mudanças climáticas, pode-se ainda acrescentar alguns novos desafios: a exigência, por parte dos clientes, de uma forma significativa e em curto espaço de tempo. Além disso, a experiência dos autores mostrou que muitos desperdícios podem ser encontrados nesta indústria, nomeadamente, elevada taxa de defeitos; desorganização; elevados tempos de espera e tempos de preparação; elevados transportes e manuseamento dos materiais e das pessoas; produção em excesso, elevados stocks, elevada taxa de retrabalho e dificuldades em entender a importância da organização numa empresa. A Figura 1 ilustra duas situações, a primeira um específico sector da ITV sem desperdícios e a segunda com desperdícios. 

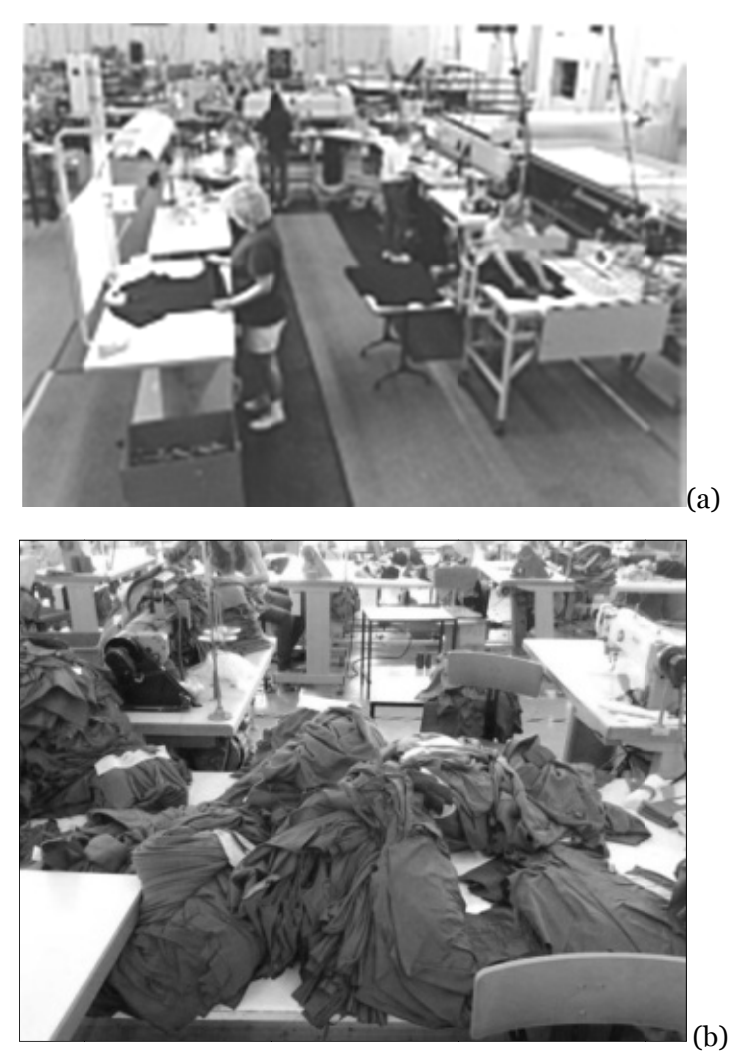

Figura 1 - Exemplo de duas situações: (a) sem e, (b) com desperdícios em específico sector da ITV Portuguesa

Outro tipo de desperdícios que deve ser também considerado, são os desperdícios ambientais. Este tipo de desperdícios é definido como o uso desnecessário ou o excesso de recursos utilizados ou substâncias libertadas para o ar, a água ou a terra que possa prejudicar a saúde humana ou o ambiente. Desperdícios ambientais podem ocorrer quando as empresas utilizam recursos para fornecer produtos ou serviços aos clientes e/ou quando os clientes usam e descartam os produtos (U.S.-EPA, 2007).

Adicionalmente, mesmo em situações de crise, é possível encontrar empresas onde os colaboradores estão acomodados a um ambiente de trabalho stressante e desagradável, em que o único objetivo, num dia normal de trabalho e de ansiedade, é ouvir a campainha anunciando o fim do dia de trabalho. Assim, é necessário o uso de técnicas, metodologias e/ou ferramentas que permitam reduzir o desperdício, mudar mentalidades e ajudar os colaboradores a encontrar algum conforto e entusiasmo no seu trabalho adotando melhores práticas de trabalho e promovendo o pensamento criativo através do envolvimento das pessoas na melhoria dos seus postos de trabalhı sendo visto como uma forma de motivação (Alves, Carvalho \& Sousa, 2012). Este potencial da capacidade humana não é normalmente explorado e consistem num outro tipo de desperdício (Liker, 2004). 
Para reduzir estes desperdícios, aumentar a produtividade e melhorar as condições de trabalho dos operadores (Maia, Alves \& Leão, 2012a), o Lean Production tem sido adotado por muitas empresas em todo o mundo como uma metodologia que oferece às empresas leanness e agilidade, e as conduz ao desenvolvimento sustentável (WCED, 1987), reduzindo o consumo de recursos naturais.

A Lean Production (LP) pode ser definida resumidamente como "... uma série de ferramentas e técnicas para a gestão de processos de uma organização. Particularmente, LP foca-se na eliminação de todas as atividades que não acrescentam valor e desperdícios provenientes dos processos. Embora as ferramentas Lean variem de implementação para implementação, o objetivo é sempre a melhoria contínua e inovação. Os projetos Lean concentram-se na eliminação ou redução de atividades que um cliente final não paga: refugo, retrabalho, inspeção, inventário, filas ou tempo de espera, transporte de materiais ou produtos, movimento excessivos e outras etapas do processo sem valor acrescentado. " (NIST, 2010).

Implementar Lean não é um processo fácil pois implica uma mudança de mentalidades, responsabilidade e competência de todos os envolvidos, sendo vital que exista um sentimento de necessidade de mudança em cada melhoria (Dahlgaard \& Dahlgaard-Park, 2006). Esta mentalidade e aprendizagem organizacional é fundamental para a melhoria contínua dos processos (Yamamoto \& Bellgran, 2010; Flinchbaugh, 2011). Para a implementação e sucesso de LP, é importante iniciá-la com o apoio da gestão de topo; caso contrário, a probabilidade de sucesso é menor. No entanto, todas as pessoas/colaboradores devem estar cientes da implementação do LP. Assim, um plano de formação profissional e comunicação será de grande importância. Não se pode esperar que a cultura da empresa de repente mude. Logo, promovendo uma comunicação aberta permitirá cativar a atenção das pessoas. Uma cultura de mudança é necessária antes de iniciar qualquer mudança, envolvendo todas as pessoas desde os gestores de topo até aos operadores do shop floor.

Com base num estudo exploratório desenvolvido pelos autores, de forma a providenciar maior conhecimento sobre como o LP está a ser implementado, ou não, na ITV Portuguesa, conclui-se que a maioria das empresas nacionais deste sector industrial desconhece a metodologia Lean. Apenas duas empresas reconhecem ter implementado LP tendo para isso recorrido a uma empresa de consultoria. Estas também afirmaram terem encontrado algumas dificuldades durante o processo de implementação do Lean, principalmente, no entendimento das ferramentas Lean, na quantificação dos benefícios e a ultrapassar atitude de resistência por parte dos colaboradores (Maia, Alves \& Leão, 2014).

Este trabalho pretende obter resposta a três perguntas de investigação (P.I. 1, P.I. 2 e P.I. 3) através da análise qualitativa de entrevistas realizadas com chefias de nível hierárquico médio/alto de uma empresa da ITV, num total de 6. Devido a restrições de disponibilidade de tempo, não foi possível incluir funcionários. Adicionalmente aos dados obtidos das entrevistas, dados recolhidos de fontes secundárias (estatísticas da empresa, entre outras) foram utilizados. Nesta fase, torna-se importante discutir e interpretar a informação recolhida nas entrevistas individuais de forma a desenvolver um conhecimento mais profundo sobre o fenómeno em estudo, elucidando linhas de investigação que eventualmente ajudem a reconhecer alguns comportamentos e que 
levem também a desenvolver uma estratégia Lean Production bem-sucedida na empresa.

\section{Metodologia e Métodos}

Para realizar um estudo mais aprofundado, foi selecionada uma empresa da ITV situada no norte de Portugal. Esta escolha baseou-se no bom relacionamento com a empresa e na sua disponibilidade para participar no estudo, assim como a receptividade e apoio na recolha de dados. A empresa selecionada ainda não implementou a metodologia LP. Este facto, porém, não é relevante para o estudo já que alguns dos seus colaboradores têm algum conhecimento sobre este conceito. Note-se que, a nível nacional, apenas cerca de $22 \%$ das empresas da ITV revelou ter algum conhecimento da metodologia e das ferramentas LP de acordo com o estudo acima referido (Maia, Alves \& Leão, 2014).

Num primeiro contato com a empresa foi agendada uma reunião com o diretor da produção/planeamento. Nessa reunião pretendeu-se explicar em detalhe o objetivo do projeto, identificando o que era necessário para o desenvolvimento do trabalho e o que era esperado por da parte da empresa. Depois de uma discussão informal, o diretor de produção reconheceu o interesse e a importância do projeto sendo de seguida, agendadas novas reuniões. A receptividade e interesse de todos os participantes foi boa. Nas visitas seguintes, diferentes procedimentos de campo foram também elaborados: reuniões, entrevistas e questionários (Maia, Alves \& Leão, 2013).

Para o inicio do trabalho foram preparadas entrevistas individuais para administração/líderes e chefias intermédias, para falar sobre a atual realidade, o que sentem sobre a empresa e as condições de trabalho. As entrevistas semiestruturadas foram realizadas com um grupo de profissionais da empresa de diferentes sectores e com diferentes experiências. Sendo as entrevistas semiestruturadas os entrevistados tiveram liberdade de expressão e facultando o seu testemunho pessoal, sobre o mesmo assunto.

\subsection{Perguntas de Investigação}

O objetivo deste estudo qualitativo foi compreender e obter resposta às três principais perguntas de investigação:

P.I. 1. Qual a necessidade de mudança?

P.I. 2. Como tenciona participar nessa mudança?

P.I. 3. Como será o comportamento/receptividade a essa mudança?

Este conjunto de perguntas de investigação conduz a um novo conjunto de perguntas complementares, tais como: "É a primeira vez que participa ou está envolvido no processo de mudança?"; "Qual a experiência de alterações anteriores?".

\subsection{Elaboração do guião da entrevista}

As entrevistas semiestruturadas foram realizadas com base num guião, incluindo os seguintes tópicos: 
1. descrição geral do projeto (para fins de contextualização);

2. caracterização do entrevistado (nome, idade, sexo, cargo, anos de serviço no cargo atual, descrição do percurso profissional, entre outros);

3. identificação dos objetivos da empresa (tópico para o administrador da empresa);

4. caracterização da população ativa do sector e percepção da consciencialização da necessidade de mudança e suas causas;

5. forma que os gestores pretendem participar na mudança (estratégias usadas);

6. porquê a mudança e como fazer essa mudança;

7. quais são os resultados esperados/resultados obtidos; e

8. sugestões.

Uma vez elaborado o guião da entrevista e selecionadas os participantes a serem entrevistados, foi obtida a permissão para fazer as entrevistas. A duração média de cada entrevista individual foi de aproximadamente 30 minutos cada. Todas as entrevistas, num total de seis, foram gravadas em áudio após autorização dos participantes e confidencialidade assegurada. Embora à partida este número possa parecer pequeno, ele corresponde praticamente ao número de sectores que a empresa está dividida e consequentemente ao número de chefias intermédias e da administração. Conforme referido por Miles \& Huberman (1994), na análise qualitativa, o tamanho da amostra é escolhida de acordo com o objetivo do estudo.

\subsection{Caraterização dos participantes}

Conforme já mencionado, as seis entrevistas realizadas, compreenderam as chefias intermédias dos sectores da empresa e a administração, a saber: o responsável pelo gabinete técnico (RGT), a responsável da qualidade (RQ), o responsável da tricotagem (RT), a responsável da remalhagem (RR), a responsável dos recursos humanos (RRH) e o responsável do planeamento da produção (PPR). Um dos entrevistados acumula dois cargos: de gestão/liderança e planeamento da produção (PPR). As principais características dos participantes deste estudo estão descritas na Tabela 1.

Tabela 1 - Caracterização dos participantes

\begin{tabular}{lllll}
\hline ID & Características & & \\
\hline & Idade & Género & $\begin{array}{l}\text { Anos na } \\
\text { empresa }\end{array}$ & Formação/Carreira profissional \\
\hline RGT & 54 & Masculino & 4 & $\begin{array}{l}\text { Técnico mecânico; com experiência em } \\
\text { manutenção de máquinas; responsável } \\
\text { pelo gabinete técnico }\end{array}$ \\
\hline RQ & 40 & Feminino & 14 & Engenharia Têxtil \\
\hline RT & 46 & Masculino & 16 & $\begin{array}{l}\text { Engenharia Mecânica; Departamento de } \\
\text { Saúde e Segurança }\end{array}$ \\
\hline RR & 32 & Feminino & 18 & Chefe de equipa; Chefe de sector \\
\hline
\end{tabular}




\begin{tabular}{lllll}
\hline ID & Características & & \\
\hline RRH & 36 & Feminino & 8 & $\begin{array}{l}\text { Engenharia Cerâmica; com experiência } \\
\text { em compras e logística; Técnica em Saúde } \\
\text { e Segurança no Trabalho }\end{array}$ \\
\hline RPP & 54 & Masculino & 14 & $\begin{array}{l}\text { Estudos Económicos (obtido no } \\
\text { estrangeiro); Diretor de Produção e } \\
\text { Distribuição }\end{array}$ \\
\hline
\end{tabular}

As primeiras percepções extraídas das entrevistas e relacionadas e que permitem responder às três perguntas de investigação (P.I. 1, P.I. 2 e P.I. 3): a consciência da necessidade de mudança, preparação para a mudança e resultados esperados, são descritas a seguir.

\section{Reflexões}

Atualmente a Indústria Têxtil Portuguesa tem uma população trabalhadora de igual número de homens e mulheres em oposição com a Indústria do Vestuário em que ainda se mantém com uma população ativa predominante feminina. A Empresa da ITV deste estudo é um exemplo disso, 80\% da população trabalhadora é feminina. No que diz respeito à formação académica, esta depende do sector. Por exemplo, no sector técnico, pela especificidade do sector e necessidade de conhecimento de línguas estrangeiras e de conhecimentos de informática, que são considerados obrigatórios, a maioria dos colaboradores tem $\mathrm{o} 11^{\mathrm{O}} / \mathbf{1 2}^{\mathrm{O}}$ ano de escolaridade. Já no sector de remalhagem como as atividades executadas são menos exigentes relativamente a conhecimentos ou formação escolar, mas requerem destreza, habilidade e prática adquirida ao longo do tempo, apresentam menor nível de formação académica $\left(6^{\circ} / 9^{\circ}\right.$ anos de escolaridade). Em relação à idade da população ativa, esta está compreendida entre os 30 a 50 anos de idade, apresentando as gerações mais jovens, maior grau de escolaridade, facto importante para favorecer a sustentabilidade das empresas.

\subsection{Consciencialização da necessidade de mudança (P.I. 1)}

Conforme o trabalho de Ford, Ford \& D'Amelio (2008), os colaboradores de uma empresa devem identificar e sentir a necessidade de mudança, para promover uma boa implementação da mudança.

No presente estudo, todos os participantes mostraram ter consciência da necessidade de mudança, realçando a importância dessa mudança.

A melhoria de desempenho da empresa foi identificado como um modo de enfrentar a concorrência e a situação real do país. Como mencionado pela responsável dos RRH:

"A mudança é sempre positiva, quando bem justificada e bem implementada."

O RQ, reconhecendo a necessidade de mudança, vai mais além referindo que para tudo existe risco associado às incertezas: 
"... a gente sempre pode aprender. (...) para o bem ou para o mal é sempre um risco, uma vez que a mudança é um risco. "

Os participantes sentem que a mudança é necessária sendo a sua preocupação a mudança organizacional, como mencionado por RGT:

"Em primeiro lugar a empresa deve-se adequar ao mercado."

Uma das razões para esta opinião poderá ser a sensação de que a mudança, que a empresa tem vinda a passar, foi forçada pela redução de efetivos e offshore da empresa, como referido por RRH:

"... Antes do processo de reestruturação, (os trabalhadores) eram muito mais, (...) permanecendo aqueles que colaboram mais e com um maior conhecimento da tarefa atribuída."

\subsection{Preparação para a mudança (P.I. 2)}

No que diz respeito à preparação para a mudança, os resultados mostram que, e segundo as percepções dos participantes no estudo, os colaboradores são sensíveis e esperam ser envolvidos no processo de mudança. Esta atitude está de acordo com estudos antecedentes, onde o diálogo e a garantia de que todas as pessoas intervenientes quando envolvidas, ajuda a diminuir a resistência à mudança (Ford, Ford \& D'Amelio, 2008). Conforme opinião mencionada pelo RR:

"Eu envolvo todos."

"(...) todos juntos podemos melhorar."

Embora a resistência à mudança seja algo natural e até de certa forma inevitável, todos os participantes foram unânimes em afirmar que suas equipas são boas e estão preparadas para a mudança.

No entanto, e de acordo com as especificidades de cada sector, pequenas diferenças foram referenciadas. De acordo com o RGT embora a equipa que lidera seja competente e bem preparada, é necessário uma constante atenção e de ser continuamente motivada e valorizar o diálogo:

"Mudar tudo (...) mudar a mentalidade (...) Primeiro, temos que dizer às pessoas para não ter medo, de falar e de expressar seus pontos de vista."

A formação profissional também foi mencionada como uma questão importante a ter em consideração para uma mudança com sucesso, como referido por RRH:

"Deve haver formação (...) tem que haver uma abertura para coisas novas."

No entanto, a mudança também poderá produzir alguma instabilidade conforme mencionado por RQ:

"(...) psicologicamente cria uma instabilidade."

Conforme acima mencionado, alguns dos participantes possuem algum conhecimento sobre o conceito Lean. Este conhecimento permitiu ao RT referir que Lean poderia ajudar no processo de mudança. 
RPP concorda que a empresa ter uma equipa muito boa não é suficiente acreditando que é necessário uma "cabeça" no comando. Esta opinião vai de encontro com alguns estudos que identificam a liderança como um factor critico e determinante no sucesso da implementação do conceito de LP nas empresas (Achanga, Shehab, Roy \& Nelder, 2006).

\subsection{Receptividade e espectativas (P.I. 3)}

É possível perceber que os acontecimentos recentes (downsizing, crise económica nos países da Europa Ocidental, ...) afetam todos e ambos, empresa e funcionários, são cautelosos e cuidadosos. A implementação de Lean para aumentar a produtividade é vista como uma boa prática, no entanto, é necessário algum cuidado para evitar implementações mal sucedidas. Em particular, a empresa sofre com a recente offshore onde a produção de alguns produtos está a ser desviada para países com mão-de-obra mais barata. Esta situação poderá ser o motivo para a abertura à mudança. Em tempos não muito longínquos, a empresa teve um shop floor com uma dimensão extraordinária, com uma produção anual de 1.00o.ooo unidades e empregava mais de 500 funcionários. Ano após ano, e com a recente offshore, algumas máquinas de grande porte e de produção, têm sido transportadas para o exterior. Nos dias de hoje, a empresa mantem as mesmas infraestruturas mas com maior espaço vazio, ocupando apenas um quarto do espaço e com uma produção de menos de 600.000 unidades por ano e empregando 162 funcionários. Nas atuais infraestruturas, é produzido os produtos de gama alta, mantendo os processos mais complexos em ambientes fechados, tais como o processo de remalhagem (Figura 2). Este processo exige operadores qualificados e bem treinados, sendo os operadores portugueses os únicos de que detêm tais características.

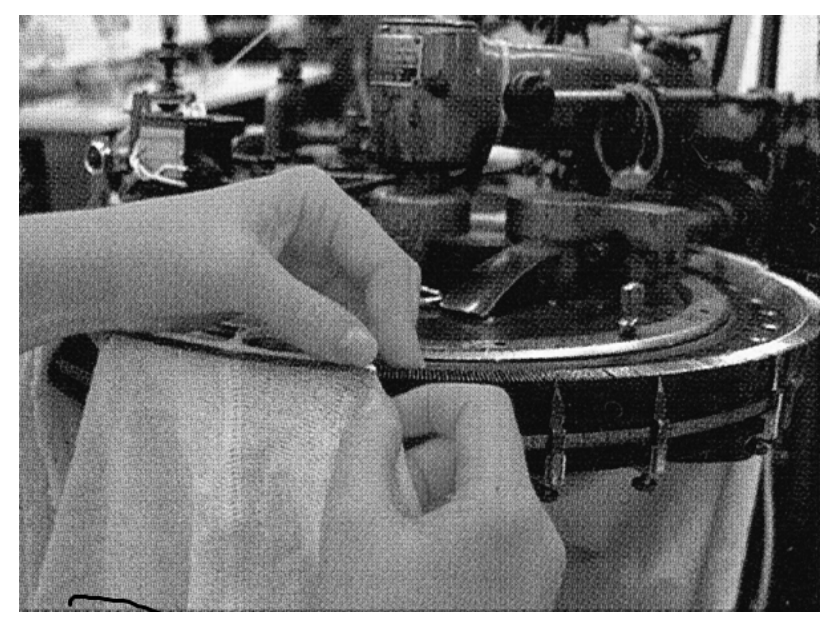

Figura 2 - Processo de Remalhagem

Ainda que, com uma menor produção, a empresa precisa mais do que nunca, de uma melhor organização de forma a agilizar os processos e a reduzir desperdícios. Estes desperdícios são devidos aos "longos" corredores e à grande infraestrutura da empresa 
que não sofreu qualquer alteração pois não acompanhou a redução do espaço necessário para a produção atual. Assim, a empresa mantém um vasto espaço físico e áreas vazias à espera de melhores dias, onde o principal desafio será atrair novos clientes/projetos ou cativar o gestor de topo a programas de internalização. Para fazer isso, é necessário uma mudança de mentalidade e de leanness para se tornar numa empresa ágil (Kidd, 1994; Gunasekaran, 2001).

\section{Identificação dos fatores que influenciam a mudança}

Dificuldade em quantificar os benefícios, dificuldade e/ou falta de conhecimentos de princípios Lean e atitudes de resistência dos colaboradores de produção, foram referidos como as dificuldades relativas à implementação de LP (Maia, Alves \& Leão, 2014).

Face às dificuldades identificadas, torna-se importante a identificação dos fatores com maior importância na mudança e perceber suas interligações.

Utilizando as reflexões obtidas através das entrevistas conduzidas, é possível identificar os fatores que influenciam a mudança positivamente como aqueles que, embora não sendo considerados como tendo uma influência negativa, podem originar alguma resistência à mudança. Figura 3 ilustra esquematicamente as possíveis interligações entre os fatores identificados.

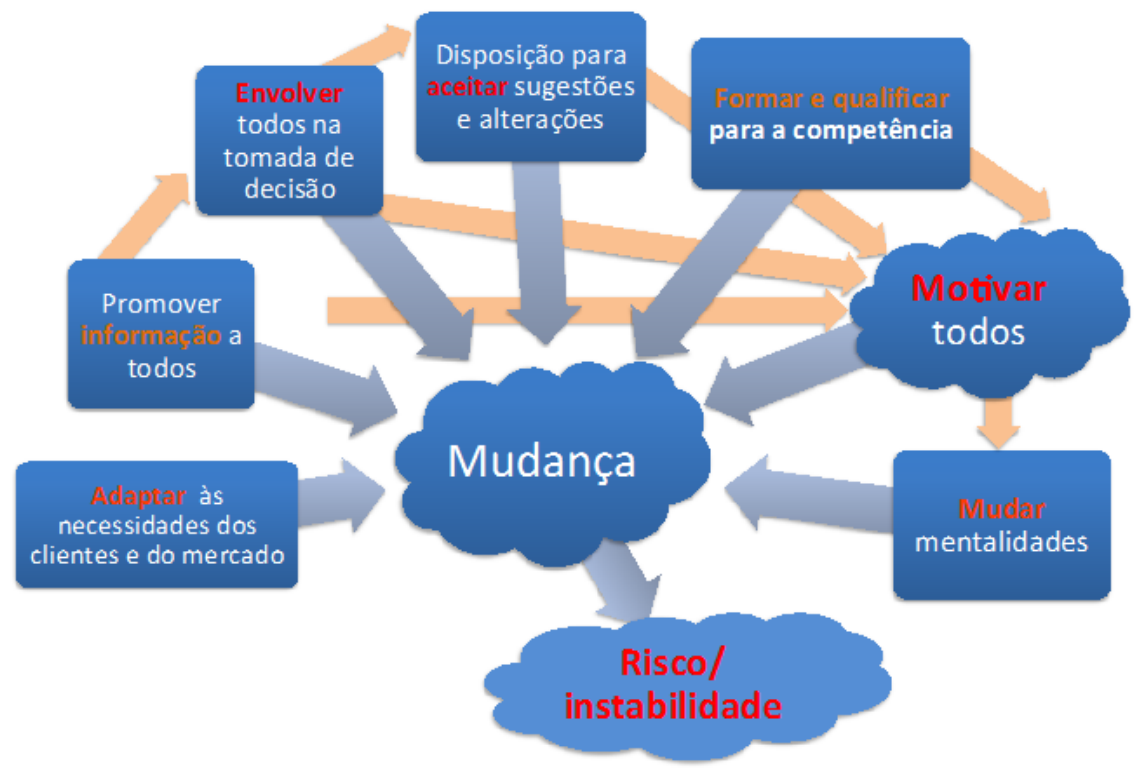

Figura 3 - Fatores identificados com influência na Mudança e suas interligações

Os fatores identificados como importantes para a mudança foram os seguintes:

- adaptar - não só às necessidades dos clientes como também do mercado, 
- informar - a informação deve ser difundida a todos,

- envolver - todos os colaboradores devem ser considerados na tomada de decisão,

- aceitar - pré-disposição para aceitar sugestões e alterações,

- formar e qualificar - todos os colaboradores para a competência,

- motivar - todos os colaboradores, e

- mudar mentalidades.

De certa forma estes factores têm uma influência positiva visto que proporcionam ou facilitam a mudança. O risco e instabilidade, por outro lado, podem ser considerados como obstáculo, criando resistência à mudança.

Todos estes fatores identificados estão também ligados entre si. Nomeadamente informar, envolver, aceitar e formar e qualificar. Um colaborador que se sinta como fazendo parte/envolvido na tomada de decisão, que se mantenha informado, que apresente sugestões e aceite alterações, de certa forma está acessível para a mudança não só de mentalidade como também de atitude para a mudança.

\section{Considerações Finais}

Neste trabalho foram analisadas entrevistas individuais conduzidas a um grupo de colaboradores de uma empresa da ITV Portuguesa sobre a necessidade de mudança e como mudar. Para melhor compreender, três principais perguntas de investigação foram definidas: "Qual a necessidade de mudança?"; "Como tenciona participar nesta mudança?"; e "Como será o comportamento/receptividade a esta mudança?".

Os participantes corresponderam às chefias de nível hierárquico médio/alto diferentes sectores de uma empresa da ITV nacional, num total de 6. Independentemente do sector, todos os participantes concordaram que a mudança é necessária, não só como forma de satisfazer as necessidades do mercado mas também para fazer frente à concorrência.

Os resultados, mostraram que os colaboradores têm um comportamento diferente no que diz respeito à resistência à mudança. Quanto mais se sentirem envolvidos nos projetos da empresa, menor a resistência à mudança. Deve ser por isso, estimulada a participação de todos nas decisões da empresa, tornando-os mais satisfeitos. Deste estudo de caso percebe-se que, mesmo considerando que a mudança pode criar alguma instabilidade, é sempre positiva, e deve ser bem justificada e explicada para uma boa implementação.

Os autores acreditam que é importante criar um bom ambiente para implementar Lean Production. Com LP é possível reduzir desperdícios e produzir mais com menos. Um dos desperdícios que a implementação LP reduz é o desperdício do esforço humano. Para isso, é necessário mudar a forma como as pessoas trabalham, por exemplo, aumentando a sua responsabilidade, motivação e satisfação (Womack, 1990; Arezes, Dinis-Carvalho \& Alves, 2010; Maia, Alves \& Leão, 2012b). 
Como trabalho futuro, destaca-se testar as diversas interligações identificadas entre os fatores com influência na mudança e motivação à mudança.

\section{Agradecimentos}

Os autores deste trabalho gostariam de expressar os seus agradecimentos à empresa da ITV e aos participantes que aceitaram o desafio de colaborar neste estudo. Os autores também agradecem aos fundos FEDER através do COMPETE e fundos nacionais da Fundação Portuguesa para a Ciência e Tecnologia (FCT) de referência do projeto: PestOE/EEI/UIo319/2014 e ao Projeto Estratégico de PEst-OE/EME/UIo252/2011.

\section{Referências}

Achanga, P., Shehab, E., Roy, R. \& Nelder, G. (2006). Critical success factors for lean implementation within SMEs, Journal of Manufacturing Technology Management, 17(4), 460-471. http://dx.doi.org/10.1108/17410380610662889

aicep Portugal Global (2014). Portugal - Ficha País. Acedido setembro 5, 2014 em http://www.portugalglobal.pt/pt/biblioteca/livrariadigital/portugalfichapais.pdf.

Alves, A. C., Carvalho, D. \& Sousa, R. (2012). Lean Production as promoter of thinkers to achieve companies' agility. The Learning Organization: an International Journal, 19(3), 219-237. http://dx.doi.org/10.1108/09696471211219930.

Arezes, P. M., Dinis-Carvalho, J. \& Alves, A. C. (2010). Threats and Opportunities for Workplace Ergonomics in Lean Environments. In Proceedings of 17th International Annual EurOMA Conference - Managing Operations in Service Economics. Sousa, R., Portela, C., Pinto, S. S. \& Correia, H. (Eds.), Universidade Católica Portuguesa, 6-9 June, 2010, Porto, Portugal.

Dahlgaard, J. J. \& Dahlgaard-Park, S. M. (2006). Lean production, six sigma quality, TQM and company culture. The TQM Magazine, 18(3), 263-281. http://dx.doi.org/10.1108/09544780610659998.

Flinchbaugh, J. (2004). Beyond Lean: Building Sustainable Business and People Success through New Ways of Thinking. Lean Learning Center. Acedido a 5 setembro, 2014 em http://www.leanlearningcenter.com/knowledge_center/ articles.cfm.

Ford, J. D., Ford, L. W. \& D'Amelio, A. (2008). Resistance to change: The rest of the Story. Academy of Management Review, 33(2), 362-377.

Gunasekaran, A. (2001). Agile Manufacturing: the 21st century competitive strategy. Elsevier Science.

Liker, J.K. (2004). The Toyota Way: 14 Management Principles from the work's greatest manufacture, McGrowHill, NY.

Kidd, P. T. (1994). Agile Manufacturing forging new frontiers. Addison Wesley Publishers. 
Maia, L. C., Alves, A. C. \& Leão, C. P. (2012a). Design of a Lean Methodology for an ergonomic and sustainable work environment in Textile and Garment Industry. ASME International Mechanical Engineering Congress and Exposition (IMECE), Volume 3, Issue PARTS A, B, and C, 1843-1852. Houston, TX, United States, 9-15 November 2012. http://dx.doi.org/10.1115/IMECE2012-89048.

Maia, L. C., Alves, A. C. \& Leão, C. P. (2012b). Do Lean Methodologies include ergonomic tools?. In Arezes, P., Baptista, J. S., Barroso, M. P., et al. (Eds.), Proceedings of International Symposium on Occupational Safety and Hygiene (SHO2012), 350-356. 9-10 fevereiro 2012, Guimarães, Portugal. ISBN: 978-97299504-9-0. WOS: 000320994300059.

Maia, L. C., Alves, A. C. \& Leão, C. P. (2013). Definition of a protocol for implementing Lean Production Methodology in Textile and Clothing case studies. ASME 2013 International Mechanical Engineering Congress and Exposition, Volume 2B: Advanced Manufacturing, Paper No. IMECE2013-64739, pp. Vo2BTo2A027; 7 pages. San Diego, California, USA, November 15-21, 2013. http://dx.doi.org/10.1115/IMECE2013-64739.

Maia, L. C., Alves, A. C. \& Leão, C. P. (2014). How Portuguese textile and Garment Industry sees the Lean Production?. Relatório interno não publicado.

Miles, M. B. \& Huberman, A. M. (1994). Qualitative Data Analysis: An Expanded Sourcebook. SAGE Publications, $2^{\text {a }}$ Edição. ISBN-10: 0803955405

NIST, The National Institute of Standards and Technology (2010). Lean, ISO, Six Sigma. Acedido setembro 5, 2014 em http://www.nist.gov/baldrige/lean_iso _sixsigma.cfm.

U.S.-EPA (2017). The Lean and Environment Toolkit. United States Environmental Protection Agency. Acedido setembro 5, 2014 em http://www.epa.gov/lean/environment/toolkits/environment/resources/LeanEvi roToolkit.pdf.

WCED (1987). Report of the World Commission on Environment and Development: Our Common Future. United Nations. Acedido setembro 5, 2014 em http://www.un-documents.net/our-common-future.pdf.

Womack, J. P., Jones, D. \& Roos, D. (1990). The machine that changed the world. New York, NY: Rawson Associates. ISBN: 0892563508

Yamamoto, Y. \& Bellgran, M. (2010). Fundamental mindset that drives improvements towards lean production. Assembly Automation, 30(2), 124-130. http://dx.doi.org/10.1108/01445151011029754. 